\title{
Electronic transport in gadolinium atomic-size contacts
}

\author{
B. Olivera, ${ }^{1}$ C. Salgado, ${ }^{2}$ J. L. Lado, ${ }^{3}$ A. Karimi, ${ }^{4}$ V. Henkel,${ }^{4}$ E. Scheer, ${ }^{4}$ J. Fernández-Rossier,,${ }^{1,5}$ \\ J. J. Palacios, ${ }^{6}$ and C. Untiedt ${ }^{1}$ \\ ${ }^{1}$ Departamento de Física Aplicada and Unidad Asociada del CSIC, Facultad de Ciencias, Universidad de Alicante, \\ San Vicente del Raspeig E-03690, Spain \\ ${ }^{2}$ Departamento de Física de la Materia Condensada, Universidad Autónoma de Madrid, Madrid E-28049, Spain \\ ${ }^{3}$ International Iberian Nanotechnology Laboratory (INL), Braga 4715-330, Portugal \\ ${ }^{4}$ Department of Physics, University of Konstanz, Universitätsstraße 10, Konstanz D-78464, Germany \\ ${ }^{5}$ QuantaLab, International Iberian Nanotechnology Laboratory (INL), Braga 4715-330, Portugal \\ ${ }^{6}$ Departamento de Física de la Materia Condensada, Condensed Matter Physics Center (IFIMAC), \\ and Instituto Nicolás Cabrera, Universidad Autónoma de Madrid, Madrid E-28049, Spain
}

(Received 15 September 2016; revised manuscript received 9 December 2016; published 8 February 2017)

\begin{abstract}
We report on the fabrication, transport measurements, and density functional theory (DFT) calculations of atomic-size contacts made of gadolinium $(\mathrm{Gd})$. Gd is known to have local moments mainly associated with $f$ electrons. These coexist with itinerant $s$ and $d$ bands that account for its metallic character. Here we explore whether and how the local moments influence electronic transport properties at the atomic scale. Using both scanning tunneling microscope and lithographic mechanically controllable break junction techniques under cryogenic conditions, we study the conductance of Gd when only few atoms form the junction between bulk electrodes made of the very same material. Thousands of measurements show that Gd has an average lowest conductance, attributed to single-atom contact, below $\frac{2 e^{2}}{h}$. Our DFT calculations for monostrand chains anticipate that the $f$ bands are fully spin polarized and insulating and that the conduction may be dominated by $s, p$, and $d$ bands. We also analyze the electronic transport for model nanocontacts using the nonequilibrium Green's function formalism in combination with DFT. We obtain an overall good agreement with the experimental results for zero bias and show that the contribution to the electronic transport from the $f$ channels is negligible and that from the $d$ channels is marginal.
\end{abstract}

DOI: 10.1103/PhysRevB.95.075409

\section{INTRODUCTION}

Quantum transport plays a key role in the electrical response of atomic scale contacts, giving rise to new phenomena differing from the bulk behavior of the different materials [1-3]. The central assumptions that allow a first guess of the conductance of an atomic scale contact are two. First, the conductance of the system is determined by the elastic transmission of the electrons at the Fermi level (Landauer formalism) and, second, the number of transmission channels that appear in the Landauer formula is determined by the chemical valence of the atoms [4].

After three decades of exploration of electronic transport in atomic scale contacts, many materials with different physical properties have been studied. The groups that are relatively well understood include noble metals, such as $\mathrm{Au}$ [5] and $\mathrm{Pt}$ [6], sp metals, such as $\mathrm{Al}[7,8]$ and $\mathrm{Zn}$ [9], ferromagnetic $3 d$ transition metals, such as $\mathrm{Fe}, \mathrm{Co}$, and $\mathrm{Ni}$ [10], superconductors, such as $\mathrm{Pb}[1,11,12]$, and even semimetals such as $\mathrm{Bi}[13,14]$. Besides, some metals like Ir [6], Pt [6], and Au [15,16] form single-stranded chains of atoms. Still, despite of all this effort, some important families remain to be covered.

In this context, while atomic contacts with $s, p$, and $d$ electrons have been widely explored, systems with partially filled $f$ shells remain pretty much an uncharted territory (with a few exceptions [17,18]). On the other hand, there has been an interest to unveil the role of magnetism in the electronic transport in atomic-sized contacts. Later attempts in $d$ materials have shown Kondo screening of the magnetic moments at such scale [10,19,20]. $f$ materials are therefore also good candidates to study the influence of the $f$ decoupled magnetic moments on the transport electrons, mainly of $s-p$, and maybe $d$ character.

$\mathrm{Gd}$ is a rare-earth metal that belongs to the lanthanide group with the electronic configuration [Xe] $4 f^{7} 5 d^{1} 6 s^{2}$. It is a trivalent metal [21] that in bulk is a strong ferromagnet with $T_{C}=293.2 \mathrm{~K}$ [22] with hexagonal close-packed (hcp) structure. It presents interesting properties, such as very high neutron absorption [23,24] and a pronounced magnetocaloric effect [25]. Regarding other types of experimental measurements on rare earths, studies of electron-magnon interaction on point contacts made of $\mathrm{Gd}$, holmium (Ho), and terbium (Tb) [26] as well as electronic structure measurements with photoelectron spectroscopy [27] have been performed. There are few experimental works about electronic transport on rare-earth atomic-size contacts. Some of them [17,18,28] reported measurements on nanocontacts made of metals such as yttrium (Y), cerium (Ce), dysprosium (Dy), and Gd by using the notched-wire mechanically controllable break junction (MCBJ) technique [2].

Concerning calculations on lanthanide materials, not much has been published for atomic-scale contacts, but their bulk properties have been widely studied. Calculations of the magnetic moment $[29,30]$ of bulk Gd compare well with the measured $7.63 \mu_{B}$ [22], where approximately $7 \mu_{B}$ come from the $4 f^{7}$ orbital. As a result, the remaining $0.63 \mu_{B}$ belong to the conduction electrons. Exchange interaction studies on $\mathrm{Gd}$ can be found elsewhere [31]. Moreover, several groups have calculated the electronic-band structure [32] from where the electronic density of states (DOS) as well as its projection on different orbitals have been inferred [33-35]. 
Here we present a combined experimental-theoretical work with two independent experimental techniques along with using density functional theory (DFT) calculations. With scanning tunneling microscopy (STM) we obtain a higher amount of statistical data than can be obtained with lithographed MCBJ, which offers samples with much higher temporal stability. DFT calculations of both electronic structure and transport properties have been carried out to give more insight into the interpretation of the experimental results.

\section{EXPERIMENTS}

Atomic-size contacts are the narrowest experimentally accessible junctions between bulk electrodes made of the same material [36] (see inset in Fig. 1). In this work we investigate nanocontacts made of pure metallic $\mathrm{Gd}$. In order to study electronic transport on nanocontacts, we use STM [37] and lithographic MCBJ [38,39] techniques independently. With both techniques we record the electrical current through
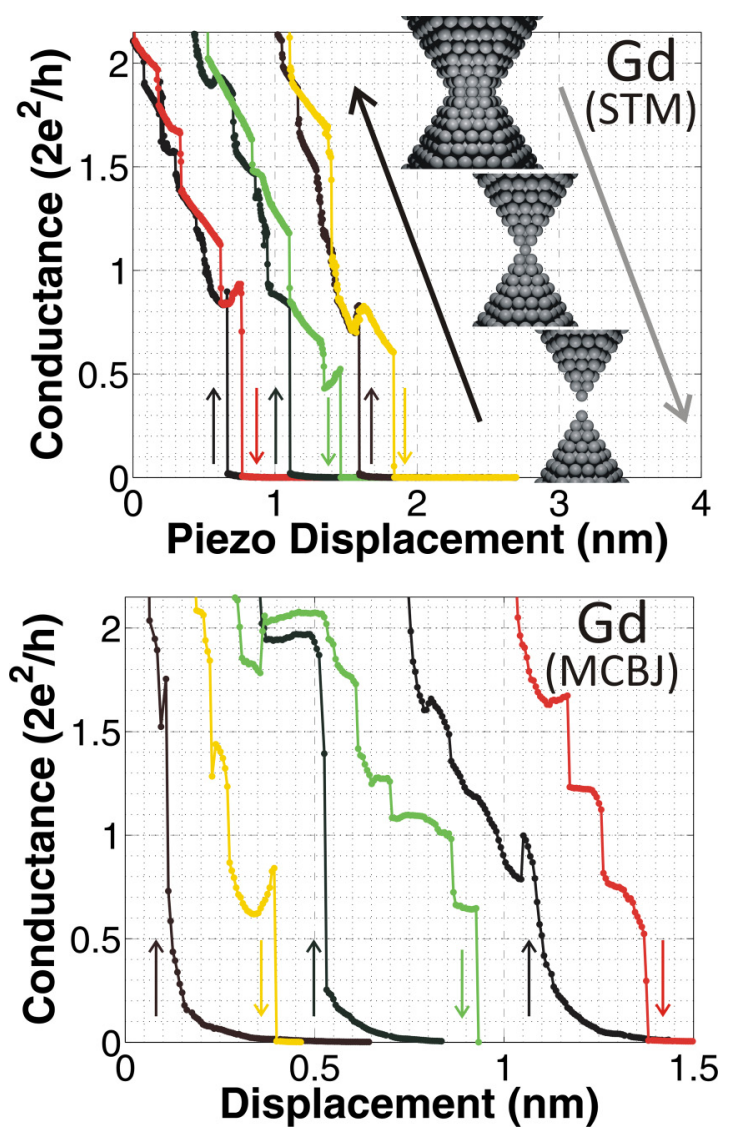

FIG. 1. Typical conductance traces for atomic-size contacts made of Gd. Bright (dark) curves stand for breaking (creating) contacts, as the arrows indicate. (Top) Measurements taken with the STM technique in equilibrium with liquid $\mathrm{He}$ bath and at $10^{-8} \mathrm{mbar}$. All traces have been taken at $100 \mathrm{mV}$ bias voltage. (Inset) Artistic representation of nanocontacts; hcp ball stacking is pictured, where balls represent atoms. (Bottom) Measurements taken with the lithographic MCBJ technique in equilibrium with liquid He bath and at $10^{-5}$ mbar. Red and green traces and their return traces have been taken at $5 \mathrm{mV}$ bias voltage; the yellow curve has been taken at $10 \mathrm{mV}$. nanocontacts under fixed applied dc bias voltage when changing the contact geometry.

We use the STM technique in contact mode and we read the current from a low-noise amplifier with a gain factor of 5. With piezoelectric materials we control the distance between bulk electrodes with atomic precision $(\sim 1 \mathrm{pm})$ under cryogenic conditions [liquid helium $(\mathrm{He})$ bath]. Samples consist of two Gd (99.9\% pure) wires of $0.5 \mathrm{~mm}$ diameter and cross shaped arranged in order to avoid multicontact locations. With this technique we build atomic contacts in a straightforward way, that is bringing into and out of contact the bulk wire-shaped electrodes by applying electrical dc sawtooth pulses to the piezoelectric materials mentioned above.

Gd gets quickly oxidized in contact with air. In order to avoid contact with environmental compounds and to preserve the purity of these materials, we use the following methods. For STM experiments, we mount samples inside a custom-made controlled atmosphere chamber. We use argon gas (99.999\% pure) as the surrounding atmosphere before closing the STM under high-vacuum conditions $\left(10^{-8} \mathrm{mbar}\right.$ reached with turbomolecular pumping). Besides, right before starting pumping, a ceramic (i.e., insulator and nonmagnetic) knife is used for scratching the outer layer of Gd wires that are afterward brought into contact. After pumping at room temperature, the STM is inserted into a bath cryostat filled with liquid He. Then, when samples reach equilibrium with the cryogenic liquid temperature, we measure the conductance as a function of distance when approaching or retracting the electrodes, so-called conductance (creating/breaking) traces.

For comparison, we use the MCBJ technique [39], in which a motor moves the pushing rod of a three-point bending mechanism with micrometric precision. This rod bends the sample from the rear side of the substrate right below the nanojunction location. The fabrication process of the latter will be explained below. The movement of the rod is reversible, so that atomic contacts can be created and broken repeatedly and the electronic transport through them is measured in the same way as with the STM technique.

In MCBJ experiments, we ensure the purity of Gd samples from the technique principle itself. As substrate we use $250-\mu \mathrm{m}$-thick gently polished bronze foil covered by a $\approx 2-\mu \mathrm{m}$-thick polyimide layer serving for planarization and electrical isolation and as sacrificial layer. Electron-beam lithography is performed in a scanning electron microscope equipped with a pattern generator to expose a polymethylmethacrylate-based two-layer resist. After development of the resist, Gd pellets with $99.9 \%$ purity are thermally evaporated from a $\mathrm{W}$ boat, which prevents alloy formation. In order to avoid possible oxide coming from pristine $\mathrm{Gd}$ pellets, we cover the substrate for the first couple of evaporated nanometers of material. The evaporation speed is adjusted to have minimal mechanical strain in the film. The sample fabrication is completed by lift off in acetone and reactive ion etching in an isotropic oxygen plasma to reduce the polyimide thickness, thereby suspending a Gd bridge of length $\approx 2 \mu \mathrm{m}$, width $\approx 100 \mathrm{~nm}$, and thickness $\approx 75 \mathrm{~nm}$. The sample is then mounted to a three-point bending mechanism anchored to a cryostat insert, pumped to a moderate high vacuum of $10^{-5}$ mbar, and cooled down to liquid He temperature. The MCBJ contact is broken for the first time when $4.2 \mathrm{~K}$ are 
reached. In this way, the few-nanometer-thick outer layer of Gd oxide at the sample protects the pure Gd nanojunction before MCBJ measurements start.

For both techniques, we record the electrical conductance as a function of distance between bulk electrodes [5], obtaining the conductance traces (see Fig. 1). We focus on the last stages before breaking the contacts into the vacuum tunnel regime and the first ones when establishing metallic atomic-size contacts. The conductance of the last (first) plateau is in the order of $\frac{2 e^{2}}{h}$, as expected for a quantum conductor with a few channels, and shows abrupt changes as a function of the electrode distance that reflect variations in the atomic configuration of the nanocontact (see Fig. 1).

In Fig. 1 we show typical conductance traces. For the STM technique (top plot), we show a series of measurements with a few seconds' space between them where deep indentations are performed. We observe that the last plateau always falls at conductance values visibly smaller than $\frac{2 e^{2}}{h}$. The same observation applies for MCBJ measurements (bottom plot). We see that the plateau shapes are negatively inclined, i.e., revealing lower conductance upon further stretching, as previously observed for some materials such as $\mathrm{Pb}$ [12], but different from the observations of other soft metals, like Au. However, some of the last plateaus reveal rising conductance upon stretching, as systematically observed, for example, in the case of $\mathrm{Al}[7,8,12]$. Both effects, the falling and the rising last plateaus, are observed with both measurement techniques and are therefore attributed to intrinsic properties of $\mathrm{Gd}$ contacts. Another observation that appears in the traces recorded with both techniques is that upon creating the contact in most cases the conductance of the first contacts is higher than the conductance of the last contact before breaking in the preceding trace. Furthermore, the backlash between breaking and creating the next contacts is somewhat larger in the MCBJ technique, presumably because of the elastic deformation of the suspended nanobridge. As a final remark, we have checked that these materials do not form long atomic chains when stretching.

With the STM technique we manage to create stable lownoise traces of conductance at a rate of about 10 traces per second. This allows us to obtain significant statistical data in a relatively brief period of time. The MCBJ technique enables mechanically more stable contacts than with the STM, but its rate of recording conductance traces is limited to about one trace per minute. We make histograms of conductance [40] out of the measured traces with STM and MCBJ (see Fig. 2).

Thousands of conductance traces with deep indentations (beyond $100 \frac{2 e^{2}}{h}$ ) are recorded along with electromigrative fast $(\approx 0.5 \mathrm{~s}) \mathrm{dc}$ pulses of $10 \mathrm{~V}$ that are applied to randomly chosen atomic-size contacts. $\frac{2 e^{2}}{h}$ is the conductance quantum, where $e$ is the elementary charge and $h$ is Planck's constant. A conductance of $100 \frac{2 e^{2}}{h}$ corresponds to a nanoconstriction with more than 100 atoms in cross section.

We have recorded conductance traces for different electrode configurations. Every configuration comes from geometrical reconstruction of bulk electrodes by strong indentation of electrodes with the STM technique (beyond $100 \frac{2 e^{2}}{h}$ ). With MCBJ, a smaller amount of data is obtained (see caption for Fig. 2); therefore, a lesser variety of traces of conductance is achieved. Different families of conductance traces for this

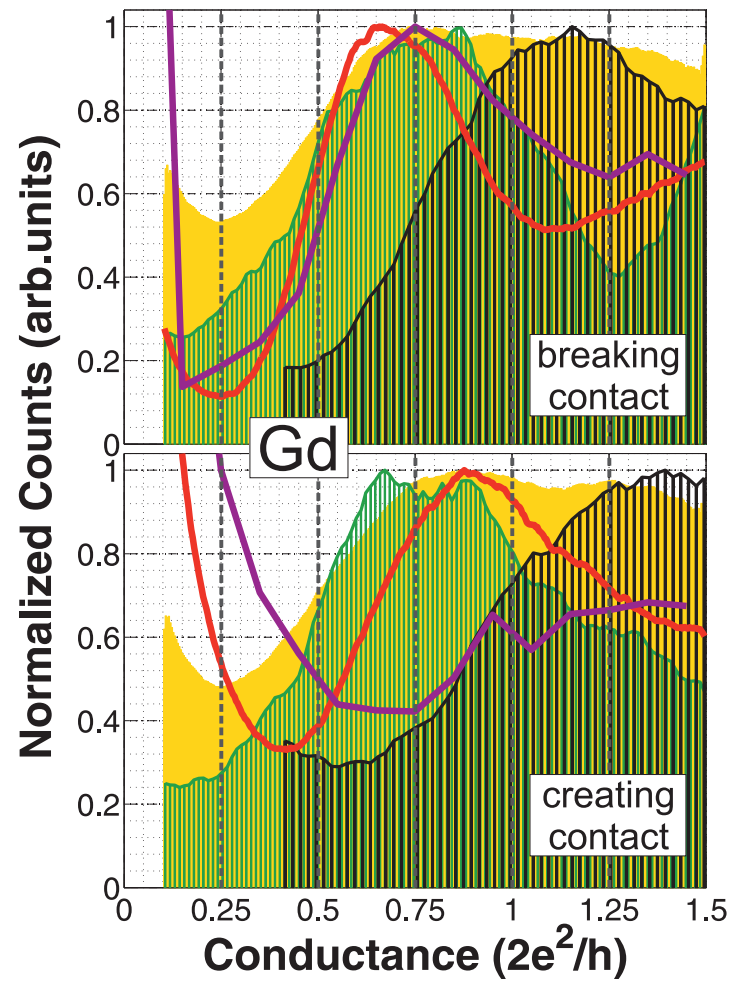

FIG. 2. Histograms of conductance calculated from conductance traces for Gd atomic-size contacts. The top (bottom) plot stands for breaking (creating) contact mode. Same colors for top and bottom plots stand for a set of traces with electrodes that have not been modified with deep indentations (i.e., less than $\approx 20 \frac{2 e^{2}}{h}$ ). The different colors (red, purple, yellow, black, green) illustrate the variability in the histograms between contacts, or for different choices of depth of indentations. All measurements have been taken with the STM technique at $100 \mathrm{mV}$ bias voltage in equilibrium with liquid He bath and $10^{-8}$ mbar, except the purple curve, that has been taken with the MCBJ technique, where a bias voltage of $10 \mathrm{mV}$ has been applied. For STM histograms, a few (from 1 to 10) thousands of traces are considered. For $\mathrm{MCBJ} \approx 500$ traces are included.

last technique are obtained, moving back the pushing rod until reaching conductances beyond $50 \frac{2 e^{2}}{h}$.

Our results agree with those of Berg et al. [28], who reported values of $(0.60 \pm 0.23) \frac{2 e^{2}}{h}$ and $(0.83 \pm 0.32) \frac{2 e^{2}}{h}$ for the last plateau right before breaking (746 curves) and first one after creating (568 curves) the contact, respectively, of Gd notchedwire MCBJs recorded at $4.2 \mathrm{~K}$. Similarly, low conductance values were also observed for Dy $\left[(0.87 \pm 0.27) \frac{2 e^{2}}{h}\right.$ from 528 breaking traces]. Reported measurements on nanocontacts made of Dy [17] showed a nontrivial change of conductance as a function of the value of the external magnetic field. We observe similar but weaker magnetostrictive effect for some lithographic Gd MCBJ samples, in our case changing from sample to sample (see Fig. S1 in the Supplemental Material [41]).

From every set of traces with electrode indentations that have not reached conductance values above $\approx 20 \frac{2 e^{2}}{h}$, we build up histograms. Some of them are shown in Fig. 2. For most well-studied metals $(\mathrm{Au}, \mathrm{Pt}, \mathrm{Ni}, \ldots)$ the position of the lowest maximum in the conductance histograms is very 

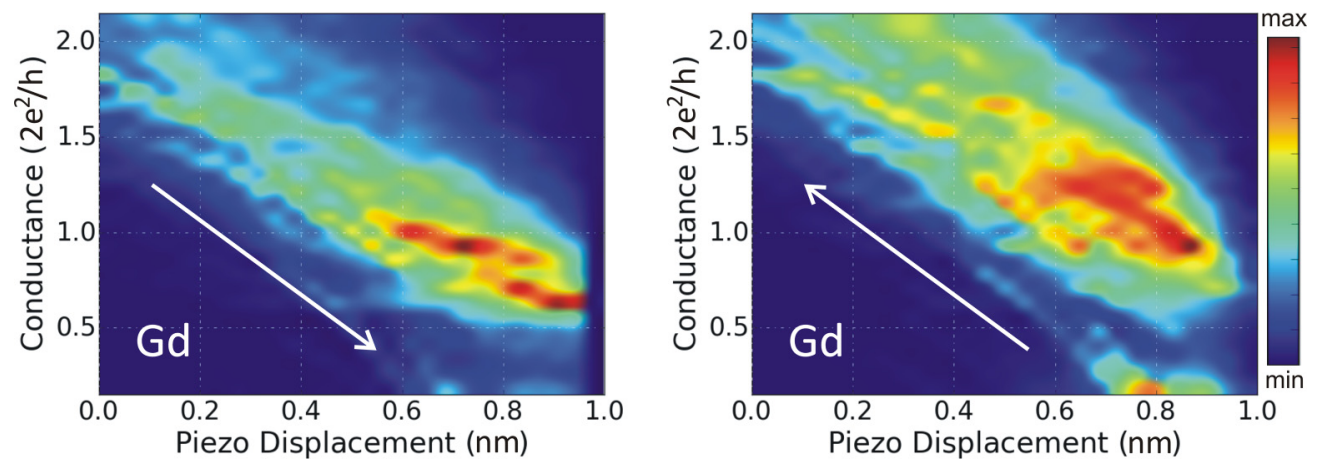

FIG. 3. (Linear color scale) Overlapped Gd conductance traces measured with STM. Breaking contact (left plot) and creating contact (right plot) situations are shown. Traces are centered to $1 \mathrm{~nm}$ when $0.01 \frac{2 e^{2}}{h}$ is reached from higher (lower) to lower (higher) conductance values for breaking (creating) contacts. Total number of traces are 2524 and 2511 for breaking and creating contact cases, respectively.

reproducible from experiment to experiment, although slight differences in the relative height and shape of the peaks have been reported $[5,6,10]$. In the case of $\mathrm{Gd}$ the positions of such histogram peaks show a lower reproducibility, which we attribute to different configurations of the electrodes. In Fig. 2 we show five independent histograms (solid lines, solid color, and vertical striped line patterns facilitate the identification of each one of them). We want to remark the strong resemblance between red (STM) and purple (MCBJ) breaking histograms. On the other hand, for MCBJ closing histograms, the first peak is located at higher conductance values, suggesting that first MCBJ contacts are thicker than first contacts created with the STM. The yellow curve shows a very wide histogram; its corresponding traces were obtained with deeper indentations, meaning that a greater random rearrangement of the electrode tips was achieved. The black curve shows higher conductance values for both breaking and creating histograms. The contacts that account for this result were poorly sharpened, as could be noticed from the high slope of the conductance vs displacement curves [42], meaning therefore, that thicker tips are considered.

In addition, the last value of conductance before breaking contacts is well below that the one observed for other magnetic metals such as, e.g., Ni [10], where a mean value of conductance of $\approx 1.5 \frac{2 e^{2}}{h}$ is found.

In order to gain further insight into the evolution of conductance traces, we have constructed intensity maps as a function of both the conductance and the displacement of the electrodes for our measured data, shown in Fig. 3. This time, we collect all the conductance traces that we have measured with STM with indentations mostly up to $20 \frac{2 e^{2}}{h}$ and plot them in a two-dimensional histogram. In order to highlight the atomic-size contact area, we center the traces at the same value of piezo displacement for a chosen conductance value (see figure caption). This way of representing data makes it possible to check the dispersion of data at the low conductance stages, unveiling the dependence of the most probable conductance on the applied strain. At the right-sided edge of the represented cloud of data a dispersion of $\approx 0.25 \frac{2 e^{2}}{h}$ is clearly apparent, one quarter less than in the case of the conductance histograms in Fig. 2. For the closing curves the highest density is observed for the conductance $(0.9 \pm 0.3) \frac{2 e^{2}}{h}$, while for the opening traces the distribution splits into two branches, one ending with a conductance of $(0.9 \pm 0.3) \frac{2 e^{2}}{h}$ at slightly shorter distance and one ending at $(0.65 \pm 0.20) \frac{2 e^{2}}{h}$ at the rightmost extremity of the cloud. This finding indicates that longer contacts with lower conductance are formed when opening. Backed up by the calculations presented in the next section, we will interpret the longer constrictions as dimeric configurations where two atoms in series form the constriction, while the shorter ones are single-atom or monomeric atomic configurations in which a single atom is surrounded by thicker electrodes on both sides. As we will discuss below, this may be unexpected, because of the presence of a $s$ band at the Fermi energy, which normally contributes with one open channel per spin.

\section{THEORY}

Density functional theory calculations are initially carried out with the linearized augmented planewaves (LAPW) code ELK. Correlations in the $f$ orbitals are treated using the DFT + U method in the Yukawa scheme [43] in the fully localized limit, to account for the strong correlations within the $f$ orbitals. No correlations are introduced in the $s, p$, and $d$ orbitals due to their delocalized nature, well accounted for by more conventional functionals. Spin-orbit coupling is treated in the noncollinear formalism. Within this framework, the bulk lattice constant matches the experimental one within $3 \%$ deviation. To gain insight into the electronic structure in the constriction, we calculate the electronic structure of one-dimensional $\mathrm{Gd}$ chains and compare the differences driven by the reduced dimensionality. Chain structures are optimized in the lattice parameter.

The magnetic structure strongly changes upon reducing the dimensionality from bulk to a one-dimensional chain (Fig. 4). Whereas the bulk structures yield a total magnetic moment of $\mu^{\text {bulk }}=7.61 \mu_{B}$, one-dimensional chains show larger moments of $\mu^{\text {chain }}=8.9 \mu_{B}$. The projected density of states reveals that, whereas bulk structures have spin polarization mainly coming from the $f$ levels plus a small contribution from $s$ ones (not shown), chains show a stronger polarization in the $d$-band manifold arising from a stronger Stoner instability (see Fig. 4). This translates into energy differences between antiferromagnetic and ferromagnetic configurations, $J=E_{A F}-E_{F E}$, in chain structures which are much larger than the ones expected for bulk. Specifically, we get $J=0.5 \mathrm{eV}$, favoring a fairly stable ferromagnetic 
(a)
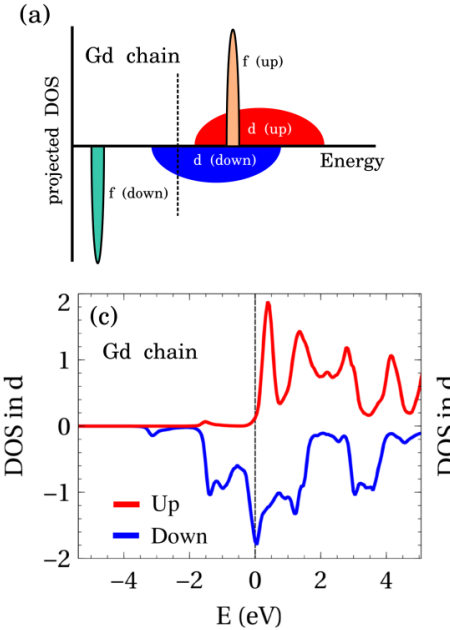

(b)
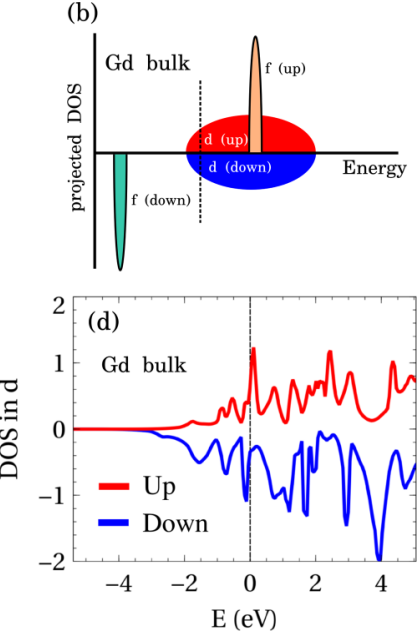

FIG. 4. Sketch of the projected DOS onto the $d$ and $f$ manifold for Gd in chain (a) and bulk (b). The $d$ manifold acquires a large spin splitting only in the chain case, while the $f$ manifold is spin polarized in both cases. Panels (c),(d) show the DOS projected onto the $d$ manifold as calculated by the first-principles full potential method for the chain (c) and bulk (d) Gd, in agreement with the sketch (a),(b).

configuration. Notice that the strong exchange coupling in chains can be understood as a consequence of the direct $d$ - $d$ exchange coupling, whereas in bulk $d$ magnetism barely appears in our calculations.

Transport calculations for $\mathrm{Gd}$ atomic contacts are also carried out. We have chosen simplified models with pyramidal forms growing along the $\langle 111\rangle$ direction for both sides of the nanocontacts (see inset in Fig. 1). The electron reservoirs, which make the nanocontact an open quantum system, are chosen to be Au electrodes. Au electrodes reduce the computational cost without introducing artifacts in the actual conductance of the model nanocontacts when these contain a large-enough number of $\mathrm{Gd}$ atoms. The results presented below correspond to the minimum number of $\mathrm{Gd}$ atoms that needs to be considered (contacts with a larger number of Gd atoms have been studied, not finding significant differences). The transport methodology is the well-known

DFT-based nonequilibrium Green's function formalism, as implemented in the package ANT.G [44-48]. This software uses the DFT functionality of GAUSSIAN09 [49] to construct the one-particle Hamiltonian of the system. This Hamiltonian constitutes the basis for the implementation of the nonequilibrium Green's function (NEGF) method through the Landauer-Keldysh formalism, which allows the simulation of open quantum systems connected to electron reservoirs.

The NEGF-DFT method implemented in ANT.G operates in the framework of linear combination of atomic orbitals (LCAO). Therefore, as a necessary first check, we need to compare with the well-grounded results of ELK. The basis "Stuttgart RSC 1997 ECP" has been used for Gd [50-52] in the LCAO-DFT calculations for chain and nanocontact structures. This basis set includes ECP (energy-consistent pseudopotentials) to describe the interaction with the core electrons. Due to the lack of a DFT $+\mathrm{U}$ implementation in the LCAO codes used, instead we make use of the hybrid functional HSE06 [53], which also captures the strongly correlated nature of the $f$ levels. In this hybrid functional developed for metals the exchange energy term is split into short-range and long-range components and the Hartree-Fock long range is neglected but compensated by the Perdew-BurkeErnzerhof long range.

We compare the results obtained in two schemes for the DOS of the one-dimensional infinite chain (see Fig. 5). The LCAO calculations are performed with the code CRYSTAL14 [54] using the same basis set and functional as for the transport calculations shown below. We note that the spinsplitting cases are slightly bigger in the LCAO method (around $20 \%$ ), which also leads a more peaked resonance structure in the DOS. These differences can be traced back to the exact exchange present in the HSE06 functional calculations, which affect the four $s, p, d$, and $f$ manifolds. In comparison, in the LAPW method, correlations included with the DFT $+U$ scheme are introduced in the localized $f$ manifold but not in the delocalized $s, p$, and $d$ manifolds. In spite of these spectral differences, the magnetic moment calculated with both methods yields a similar value. Nevertheless, differences in the character of the electrons around the Fermi energy, which dominate the electronic transport, may yield different
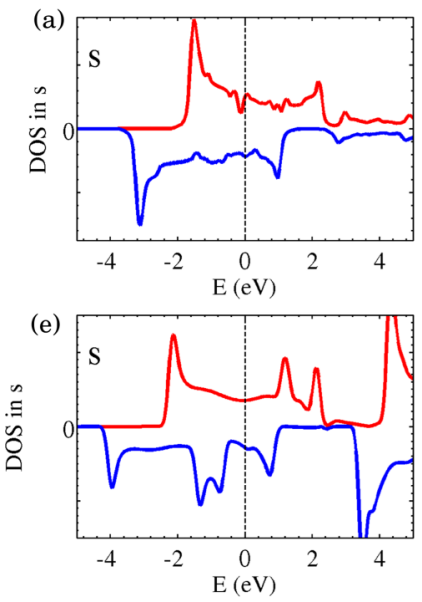
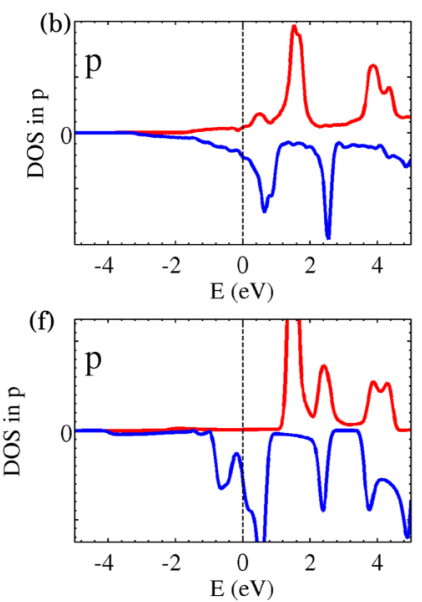
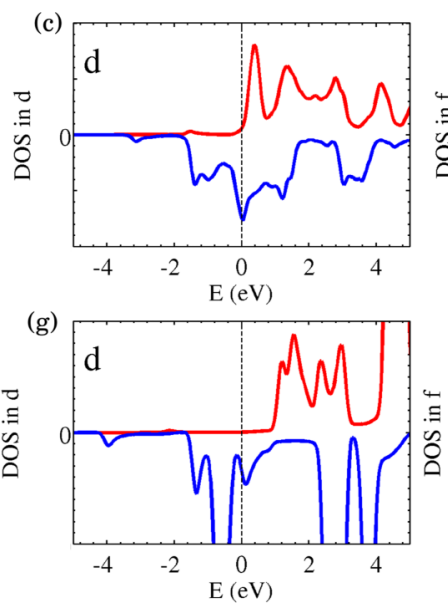
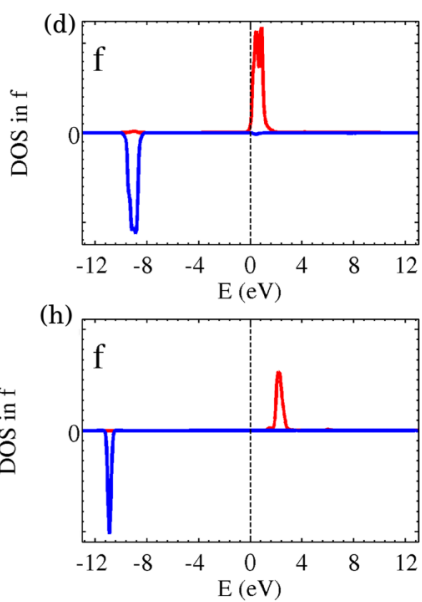

FIG. 5. Spin-resolved density of states of Gd in chain structures calculated with ELK (LAPW) projected onto the manifolds $s$ (a), $p$ (b), $d(\mathrm{c})$, and $f(\mathrm{~d})$. Same cases calculated with CRYSTAL14 (LCAO) $[s(\mathrm{e}), p(\mathrm{f}), d(\mathrm{~g})$, and $f(\mathrm{~h})]$. 

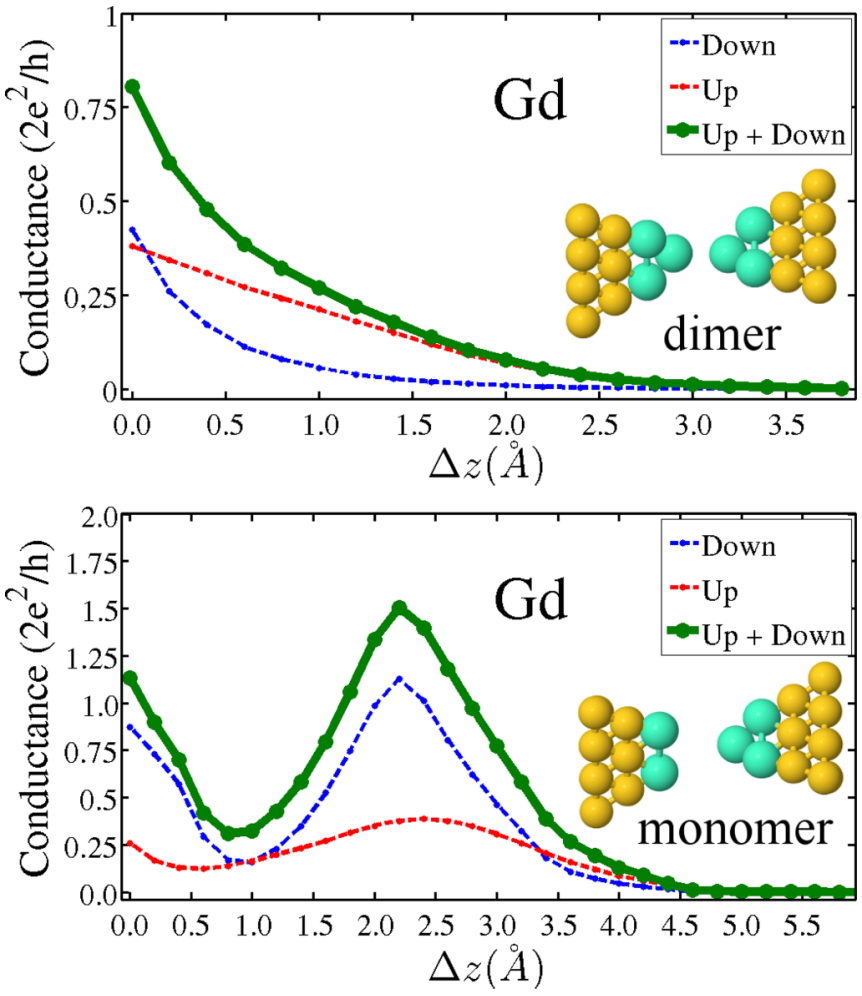

FIG. 6. Gd $\langle 111\rangle$ nanocontact calculated conductance-distance characteristic. (Top) Two atomically sharp tips in the $\langle 111\rangle$ direction forming a dimeric contact. The distance $\Delta z$ equals 0 when the distance between both tip apex atoms equals the nearest-neighbor distance in bulk. (Bottom) An atomically sharp tip in the $\langle 111\rangle$ direction against a blunt $\langle 111\rangle$ one. Both tips touch to form a monomeric contact. The distance $\Delta z$ equals 0 when the distance between the tip apex atom in the atomically sharp tip and each tip apex atom in the blunt one equals the nearest-neighbor distance in bulk.

conductance values. By considering different functionals (with and without exact exchange) we have concluded that these differences are not significant in this regard.

In the following we present the results obtained for the conductance in the LCAO scheme using the HSE06 functional. The calculated conductance-distance characteristics for $\mathrm{Gd}$ nanocontacts are shown in Fig. 6 for both monomer and dimer configurations (see insets in Fig. 6). As anticipated in the discussion of the experimental results, monomer and dimer configurations are expected to form when breaking the contacts, while in most of the cases only monomers are expected to appear when forming the contacts. The piezo displacement is simulated by opposite displacement of the two tips, while keeping their atomic structure intact. Thus, we do not make any distinction between breaking and creating contacts, although a small difference in the average atomic bond distance is expected between the two processes if relaxation were allowed. Relaxation would also permit to simulate the plasticity effects (jumps in conductance), as seen in this type of experiment. This is, however, computationally too costly since a large number of $\mathrm{Gd}$ atoms are required and beyond the scope of our discussion here.

As their periodic counterparts (bulk and chains), Gd nanocontacts show a purely ferromagnetic behavior all along the breaking process. Antiparallel magnetic configurations (between the two tips) show smaller conductance values, but these magnetic states have a higher energy and tend to relax into the ferromagnetic ones. The current is spin-polarized with a dominant contribution from the minority channel (spin-up here in red) for stretched dimeric contacts and from the majority one (spin-down in blue) for monomeric contacts. The calculated total conductance at bulk near-neighbor distance between tip apex atoms (or zero displacement, $\Delta z=0$ ) is $0.80 \frac{2 e^{2}}{h}$ for the dimer and $1.15 \frac{2 e^{2}}{h}$ for the monomer contacts. Both values, along with the ones nearby for small displacements (representing actual stretched contacts) fall within their tentatively assigned experimental bright spots seen in Fig. 3. Notice that, due to lack of relaxation in our calculations, longer displacements may not represent actual atomic configurations since sudden plastic deformations must occur. Even so, for the monomer configuration we obtain an increase of conductance as the tip-tip distance increases, which is of purely electronic origin and could be tentatively related to the one often observed in the experiments [see bottom plot at Fig. 6].

A deeper insight into the electronic nature of transport can be revealed by analyzing the nature of the eigenchannels [48] involved in the conductance. In Fig. 7 we plot the conductance of the spin-up and spin-down dominant eigenchannels for three representative examples. We have chosen (a) a dimer contact at a displacement of $\Delta z=1.0 \AA$ and (b) a monomer contact at a displacement of $\Delta z=1.0 \AA$ and (c) $\Delta z=2.2 \AA$. In general, the eigenchannels do not show a dominant $s$ character. For case (a) they display mostly a $p_{z}$ character (minority) or $s p_{z}$ character (majority). In the monomer case, in addition to the $s p_{z}$ hybridization [7,12], the transition from $s p_{z}$-like eigenchannels at smaller displacements [Fig. 7(b)] to an eigenchannel with a strong $d$ character for majority spins [Fig. 7(c)] at larger displacements seems to also play a role in the change of the slope of the conductance traces, as seen in Fig. 6 (bottom plot). No direct contribution from $f$ orbitals appears in transport, apart from enhancing the spin polarization in the other subshells, as expected from their localized nature. We finally emphasize that the previous analysis relies on the assumption that the LCAO + HSE (Heyd-Scuseria-Ernzerhof) method properly captures the electronic structure around the Fermi energy for the rare-earth compound, which could change using other DFT schemes. Therefore, the accuracy of our method is subject to verification by more sophisticated ones, capable of capturing simultaneously the metallic behavior and strongly localized states in these rare-earth chains.

\section{CONCLUSIONS}

We have carried out electrical-current transport measurements on atomic-size contacts made of $\mathrm{Gd}$ under cryogenic conditions. Unlike the case of $3 d$ ferromagnetic materials and despite the $d^{1}$ valence of $\mathrm{Gd}$, their single-atom conductance is typically smaller than $\frac{2 e^{2}}{h}$. This might be at first sight surprising, because in both cases there is a wide $s$ band at the Fermi energy, which normally provides a highly transmissive channel and which, along with an additional contribution coming from the $d$ channels, may give conductance values above $\frac{2 e^{2}}{h}$. However, in the case of Gd the transmission of all 

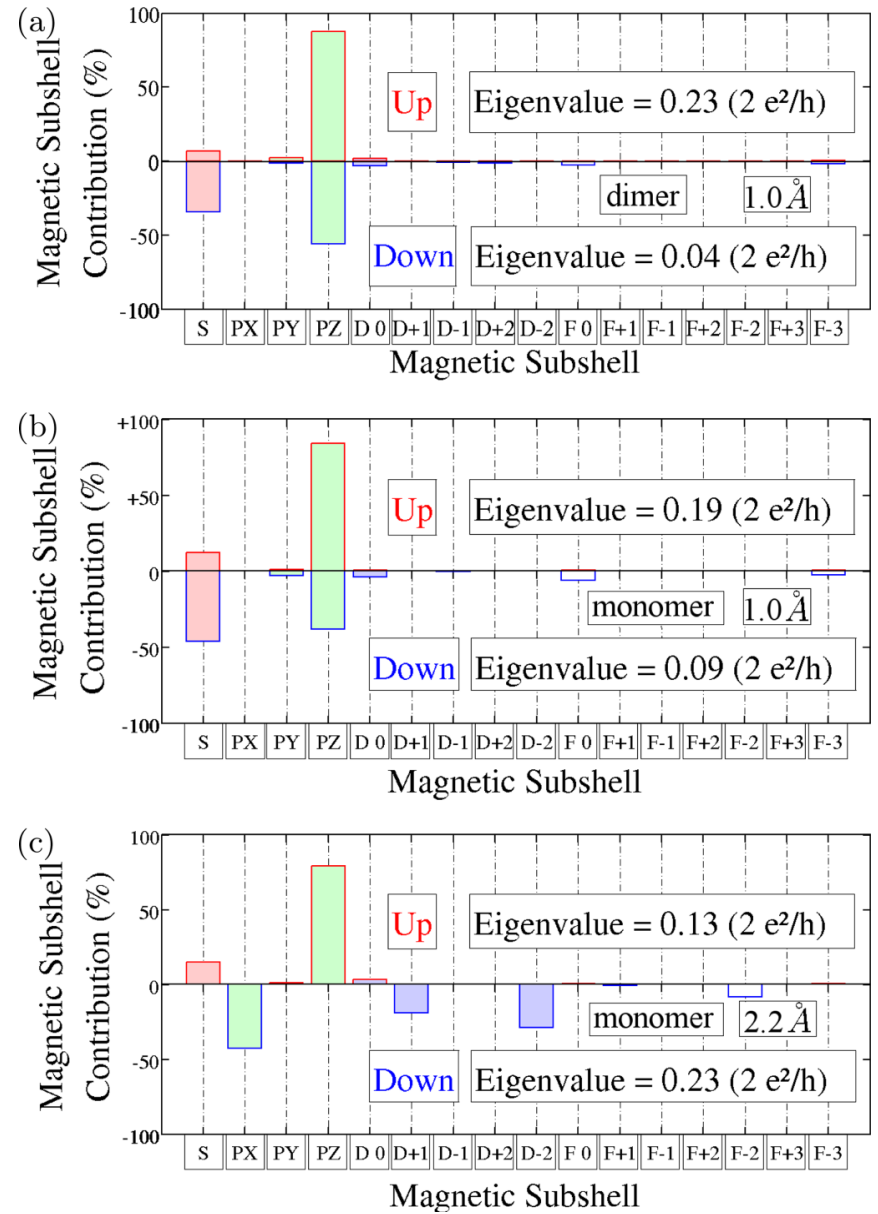

FIG. 7. (a) Spin-resolved principal eigenchannel projected onto all the magnetic shells of a Gd dimeric nanocontact at a tip-tip displacement of $1.0 \AA$; (b) and (c) the same but for a monomeric contact at tip-tip displacement of $\Delta z=1.0 \AA$ and $\Delta z=2.2 \AA$, respectively. "Up" electrons correspond to the "minority" spin component, while "down" electrons correspond to the "majority" one.

channels is reduced presumably to the position of the Fermi energy close to the edges of the respective bands. The results are reproducible for both STM and MCBJ measurements in many details: lengths of plateaus, shapes of plateaus, position and height of histogram maxima, and differences between creating and breaking curves.
Our DFT calculations show that, apart from the $f$ magnetism, the dimensionality reduction of the nanocontacts creates a spin splitting in the $d$-band manifold, in comparison with bulk structures where the $d$-band manifold remains nearly unpolarized. Using the nonequilibrium Green's function formalism within the LCAO + HSE06 scheme we generically obtain conductance values smaller than $\frac{2 e^{2}}{h}$, with differences between monomer and dimer configurations. The analysis of the eigenchannels shows that this is due to a hybridization of the $s$ and $p_{z}$ channels, which apparently reduces the conductance of a pure $s$ channel. This is also in line with the increasing conductance on the last plateau as the electrodes are pulled apart, which has also been observed in Al atomic contacts and for which the $s p_{z}$ hybridization is also known to play a role. In the case of $\mathrm{Gd}$, the $d$ orbitals also seem to play a role in this conductance rise. Finally, our zero-bias measurements do not seem to be strongly influenced by the large $f$ local magnetic moments, mainly because $f$ electrons do not participate in conduction. At finite bias, inelastic excitation of spin waves, that certainly involve the $f$-magnetic moments, might play a role.

\section{ACKNOWLEDGMENTS}

B.O., C.S., J.F.R., J.J.P., and C.U. acknowledge financial support by MEC-Spain (Grant No. FIS2013-47328-C2 and MAT2016-78625-C2) and the Generalitat Valenciana under Grant No. PROMETEO/2012/011. C.S. and J.J.P. acknowledge the EU structural funds and the Comunidad de Madrid under NANOFRONTMAG-CM program Grant No. S2013/MIT-2850. C.S. and J.J.P. also acknowledge the computer resources and assistance provided by the Centro de Computación Científica of the Universidad Autónoma de Madrid and the RES. J.L.L. and J.F.R. acknowledge Marie Curie ITN SPINOGRAPH FP7 under REA Grant Agreement No. 607904-13. B.O. acknowledges financial support by MECSpain (Grant No. FIS2010-21883-C02-01) under brief stays abroad scholarship. J.L.L. acknowledges the hospitality of the Departamento de Física Aplicada of the University of Alicante. We gratefully acknowledge helpful discussion with C. Sürgers and $\mathrm{M}$. Fonin regarding the material properties of rare-earth metals and for providing the Gd material. We thank C. Sabater, F. Strigl, and H. Ballot for experimental help.
[1] A. I. Yanson, Atomic chains and electronic shells: Quantum mechanisms for the formation of nanowires, Ph.D. thesis, Universiteit Leiden, 2001.

[2] N. Agraï, A. Levy Yeyati, and J. M. van Ruitenbeek, Phys. Rep. 377, 81 (2003).

[3] Y. G. Naidyuk and I. K. Yanson, Point-Contact Spectroscopy (Springer Science \& Business Media, New York, 2005), Vol. 145.

[4] E. Scheer, N. Agraït, J. C. Cuevas, A. Levy Yeyati, B. Ludoph, A. Martín-Rodero, G. Rubio Bollinger, J. M. van Ruitenbeek, and C. Urbina, Nature (London) 394, 154 (1998).
[5] N. Agraï, J. G. Rodrigo, and S. Vieira, Phys. Rev. B 47, 12345 (1993).

[6] R. H. M. Smit, C. Untiedt, A. I. Yanson, and J. M. van Ruitenbeek, Phys. Rev. Lett. 87, 266102 (2001).

[7] E. Scheer, P. Joyez, D. Esteve, C. Urbina, and M. H. Devoret, Phys. Rev. Lett. 78, 3535 (1997).

[8] D. Sánchez-Portal, C. Untiedt, J. M. Soler, J. J. Sáenz, and N. Agraï, Phys. Rev. Lett. 79, 4198 (1997).

[9] M. Häfner, P. Konrad, F. Pauly, J. C. Cuevas, and E. Scheer, Phys. Rev. B 70, 241404 (2004). 
[10] M. R. Calvo, J. Fernández-Rossier, J. J. Palacios, D. Jacob, D. Natelson, and C. Untiedt, Nature 458, 1150 (2009).

[11] M. Müller, C. Salgado, N. Néel, J. J. Palacios, and J. Kröger, Phys. Rev. B 93, 235402 (2016).

[12] J. C. Cuevas, A. Levy Yeyati, A. Martín-Rodero, G. R. Bollinger, C. Untiedt, and N. Agraï, Phys. Rev. Lett. 81, 2990 (1998).

[13] C. Sabater, D. Gosálbez-Martínez, J. Fernández-Rossier, J. G. Rodrigo, C. Untiedt, and J. J. Palacios, Phys. Rev. Lett. 110, 176802 (2013).

[14] H.-F. Pernau, T. Pietsch, and E. Scheer, J. Phys.: Condens. Matter 26, 474203 (2014).

[15] A. I. Yanson, G. Rubio Bollinger, H. E. van den Brom, N. Agraït, and J. M. van Ruitenbeek, Nature (London) 395, 783 (1998).

[16] H. Ohnishi, Y. Kondo, and K. Takayanagi, Nature (London) 395, 780 (1998).

[17] M. Müller, R. Montbrun, M. Marz, V. Fritsch, C. Sürgers, and H. v. Löhneysen, Nano Lett. 11, 574 (2010).

[18] S. N. Jammalamadaka, S. Kuntz, O. Berg, W. Kittler, U. M. Kannan, J. A. Chelvane, and C. Sürgers, Sci. Rep. 5, 13621 (2015).

[19] V. Madhavan, W. Chen, T. Jamneala, M. F. Crommie, and N. S. Wingreen, Science 280, 567 (1998).

[20] M. R. Calvo, D. Jacob, and C. Untiedt, Phys. Rev. B 86, 075447 (2012).

[21] K. A. Gschneidner, L. Eyring, and G. H. Lander, Handbook on the Physics and Chemistry of Rare Earth (North-Holland, 1978), Vol. 1, pp. 1-894.

[22] H. E. Nigh, S. Legvold, and F. H. Spedding, Phys. Rev. 132, 1092 (1963).

[23] R. E. Lapp, J. R. VanHorn, and A. J. Dempster, Phys. Rev. 71, 745 (1947).

[24] G. Leinweber, D. P. Barry, M. J. Trbovich, J. A. Burke, N. J. Drindak, H. D. Knox, R. V. Ballad, R. C. Block, Y. Danon, and L. I. Severnyak, Nucl. Sci. Eng. 154, 261 (2006).

[25] K. A. GschneidnerJr, V. K. Pecharsky, and A. O. Tsokol, Rep. Prog. Phys. 68, 1479 (2005).

[26] A. I. Akimenko, A. B. Verkin, N. M. Ponomarenko, and I. K. Yanson, Physica B+C (Amsterdam) 107, 369 (1981).

[27] U. Bovensiepen, J. Phys.: Condens. Matter 19, 083201 (2007).

[28] O. Berg, Elektrischer transport durch nanokontakte von seltenerd-metallen, Ph.D. thesis, KIT Scientific Publishing, 2014, Vol. 10.

[29] R. Ahuja, S. Auluck, B. Johansson, and M. S. S. Brooks, Phys. Rev. B 50, 5147 (1994).

[30] P. Kurz, G. Bihlmayer, and S. Blügel, J. Phys.: Condens. Matter 14, 6353 (2002).

[31] D. A. Goodings, Phys. Rev. 127, 1532 (1962).

[32] W. M. Temmerman and P. A. Sterne, J. Phys.: Condens. Matter 2, 5529 (1990).
[33] I. Turek, J. Kudrnovský, G. Bihlmayer, and S. Blügel, J. Phys.: Condens. Matter 15, 2771 (2003).

[34] C. Santos, W. Nolting, and V. Eyert, Phys. Rev. B 69, 214412 (2004).

[35] C.-G. Duan, R. F. Sabirianov, W. N. Mei, P. A. Dowben, S. S. Jaswal, and E. Y. Tsymbal, J. Phys.: Condens. Matter 19, 315220 (2007).

[36] J. K. Gimzewski and R. Möller, Phys. Rev. B 36, 1284 (1987).

[37] G. Binnig, H. Rohrer, C. Gerber, and E. Weibel, Phys. Rev. Lett. 49, 57 (1982).

[38] J. Moreland and J. W. Ekin, J. Appl. Phys. 58, 3888 (1985).

[39] J. M. van Ruitenbeek, A. Alvarez, I. Piñeyro, C. Grahmann, P. Joyez, M. H. Devoret, D. Esteve, and C. Urbina, Rev. Sci. Instrum. 67, 108 (1996).

[40] A. I. Yanson and J. M. van Ruitenbeek, Phys. Rev. Lett. 79, 2157 (1997).

[41] See Supplemental Material at http://link.aps.org/supplemental/ 10.1103/PhysRevB.95.075409 for a figure illustrating the magnetostrictive effect for some lithographic Gd MCBJ samples.

[42] C. Untiedt, G. Rubio, S. Vieira, and N. Agrait, Phys. Rev. B 56 , 2154 (1997).

[43] F. Bultmark, F. Cricchio, O. Grånäs, and L. Nordström, Phys. Rev. B 80, 035121 (2009).

[44] J. J. Palacios, A. J. Pérez-Jiménez, E. Louis, and J. A. Vergés, Phys. Rev. B 64, 115411 (2001).

[45] J. J. Palacios, A. J. Pérez-Jiménez, E. Louis, E. SanFabián, and J. A. Vergés, Phys. Rev. B 66, 035322 (2002).

[46] E. Louis, J. A. Vergés, J. J. Palacios, A. J. Pérez-Jiménez, and E. San Fabián, Phys. Rev. B 67, 155321 (2003).

[47] D. Jacob and J. J. Palacios, J. Chem. Phys. 134, 044118 (2011).

[48] D. Jacob and J. J. Palacios, Phys. Rev. B 73, 075429 (2006).

[49] M. J. Frisch, G. W. Trucks, H. B. Schlegel, G. E. Scuseria, M. A. Robb, J. R. Cheeseman, G. Scalmani, V. Barone, B. Mennucci, G. A. Petersson et al., Gaussian 09, revision D. 01 (Gaussian, Inc., Wallingford, CT, 2009), Vol. 4.

[50] A. Bergner, M. Dolg, W. Küchle, H. Stoll, and H. Preuß, Mol. Phys. 80, 1431 (1993).

[51] M. Kaupp, P. v. R. Schleyer, H. Stoll, and H. Preuss, J. Chem. Phys. 94, 1360 (1991).

[52] M. Dolg, H. Stoll, H. Preuss, and R. M. Pitzer, J. Phys. Chem. 97, 5852 (1993).

[53] J. Heyd and G. E. Scuseria, J. Chem. Phys. 121, 1187 (2004).

[54] R. Dovesi, R. Orlando, A. Erba, C. M. Zicovich-Wilson, B. Civalleri, S. Casassa, L. Maschio, M. Ferrabone, M. De La Pierre, P. D'Arco et al., Int. J. Quantum Chem. 114, 1287 (2014). 